\title{
Deriving a Flexible Mixed Demand System: The Normalized Quadratic Model
}

\author{
GianCarlo Moschini and Pier Luigi Rizzi
}

\begin{abstract}
This article deals with the specification of a locally flexible and theory-consistent system of mixed demand functions, a framework that allows for a rich set of possibilities about what is assumed as exogenous in a demand model. A coherent mixed demand system is derived by using the restricted expenditure function typically studied in the related area of rationed demands. The method is implemented by a new normalized quadratic (NQ) parameterization of the restricted expenditure function. The resulting NQ mixed demand system is illustrated with an application to a nine-good model of the Italian demand for vegetables.
\end{abstract}

Key words: demand systems, duality, flexible functional forms, mixed demands.

A major thrust of the system-wide approach to empirical demand analysis has been to specify models that are integrable into well-behaved preferences. The restrictions of consumer theory help, at the estimation stage, to reduce the number of parameters to be estimated and thus increase efficiency, but adherence to theory also permits meaningful use of the resulting estimates for welfare and policy analysis. The earlier work of Stone (1954), leading to the linear expenditure system (LES), was subsequently extended by models that capture more general and flexible representations of preferences, such as the translog model of Christensen, Jorgenson, and Lau (1975), the differential (Rotterdam) model of Theil (1975), the almost ideal (AI) demand system of Deaton and Muellbauer (1980), the quadratic AI demand system (Banks, Blundell, and Lewbel 1997), and the semiflexible AI demand system (Moschini 1998).

A common feature of these specifications is to represent quantity demanded as a function of market prices and total expenditure (income for short). Whereas this approach corresponds directly to the usual formulation of the individual consumer problem, its use within an econometric model requires some additional identifying assumptions-essentially, what is to be assumed as exogenous or predetermined. The standard specification of demand models

GianCarlo Moschini is professor and the Pioneer Chair in Science and Technology Policy, Department of Economics, Iowa State University. Pier Luigi Rizzi is professor, Dipartimento di Economia Politica, Università di Siena, Italy. with quantities as the dependent variable assumes that prices are predetermined. That is, if one thinks of the data at hand as the outcome of a market equilibrium model, the implicit assumption is that supply functions are perfectly elastic so that demands adjust to clear the market. While this condition may hold for market data in some situations (e.g., tradeable goods for a small open economy), it obviously is not universal. Indeed, Geary (1948-1949), in his derivation of the utility function underlying the LES, noted that "From the regression viewpoint, however, it would be equally logical to regard prices as dependent variables and quantities as independent variables..." This view has been occasionally implemented in terms of inverse demand systems, as in the (inverse) translog (Christensen, Jorgenson, and Lau 1975), the (inverse) Rotterdam model (Theil 1975, 1976; Barten and Bettendorf 1989), the linear inverse demand system (Moschini and Vissa 1992; Eales and Unnevehr 1994), and the inverse normalized quadratic (NQ) (Holt and Bishop 2002).

The choice of which variables to assume as predetermined in empirical demand models has nontrivial implications. To illustrate, if a direct demand system is specified when in fact an inverse demand specification is called for, then the duality between direct and inverse demand systems implied by consumer theory means that, for nontrivial preferences, the direct demand system will be affected by a nonlinear errors-in-variables problem. For such a case it is notoriously difficult to obtain estimators that are consistent. In fact, 
instrumental variable estimators are also inconsistent (Amemiya 1985), and their application in standard system estimation is bound to give inconsistent estimates of the underlying preference parameters (Moschini 2001). Unlike other instances of errors-in-variables in demand models (e.g., Lewbel 1996), however, the question in our setting is not about a suitable estimation technique. Rather, the question is one of choosing the appropriate assumption on what to take as predetermined in order to identify the underlying preference parameters. Given that, a mixed demand system approach provides an appealing framework of analysis.

In mixed demand functions, first analyzed by Samuelson (1965), the prices of some goods and the quantity of all the others are predetermined, so that some quantities and some prices adjust to clear the market. This class of models has obvious econometric appeal for the purpose of estimating demand behavior, because it encompasses a spectrum of possibilities between the polar cases of direct and inverse demand functions. Hence, mixed demands allow for a much richer set of options about what is to be assumed as exogenous or predetermined, which permits the identifying assumptions of demand models to be tailored to the nature of the data at hand. Despite this attractive attribute, mixed demand functions have received comparatively little attention in applied studies. Moschini and Rizzi (2006) derive and estimate a mixed demand system for the special case of Stone-Geary preferences. More general representations of mixed demand systems have essentially been confined to the Rotterdam specification. Barten (1992) appealed to mixed demand to illustrate the choice of which variables to assume as exogenous but actually estimated a standard Rotterdam model, while taking into account the endogeneity of some of the prices in formulating the likelihood function. Moschini and Vissa (1993) and Brown and Lee (2006), by contrast, formulated and estimated true differential mixed demand systems. $^{1}$

A possible explanation for the paucity of applications of mixed demand systems resides in the specific difficulties that arise in this context. That is, in Samuelson's (1965) formulation,

\footnotetext{
${ }^{1}$ Mixed demands also turn up in environmental economics (e.g., Cunha-e-Sá and Ducla-Soares 1999), although the setting there is more appropriately one of rationed demands (the goods in fixed supply are public goods and do not enter the consumer's budget constraint).
}

knowledge of both direct and indirect utility functions is required to characterize the demand properties. This means that many commonly used flexible functional forms - such as the translog indirect utility function, or the PIGLOG cost function of AI systems - cannot be used to specify a mixed demand system because these flexible functional forms do not have a closed-form dual representation. That is why the only flexible true mixed demand system that has been proposed to date relies on approximating the mixed demand equations directly through a differential approach. Even though such a Rotterdam-type mixed demand system is of considerable interest (e.g., Matsuda 2004), for some applications (such as welfare analysis) it may be desirable to have an exact parametric representation of preferences.

With this article we hope to advance the applicability of mixed demand systems in an empirical setting by making three main contributions. First, we briefly review the theoretical framework of mixed demands that makes it explicit why the procedure used in numerous applications of direct and inverse demand systems is not particularly useful. Second, we suggest a new approach to specifying a mixed demand system, based on the restricted expenditure function used in the related area of rationed demand (Gorman 1976; Neary and Roberts 1980). Mixed demands and rationed demands share important similarities, but a major difference is that for the latter some markets do not clear. In the case of mixed demands, on the other hand, the virtual prices of the quantity-predetermined goods do enter the budget constraint, so that it is in principle possible to solve for the mixed utility function implied by the restricted expenditure function and thus derive integrable mixed demand equations. We identify a class of restricted cost functions for which an explicit solution of the mixed demand equations is possible. Third, to make the approach operational, we develop a new NQ parameterization that is locally flexible, and that can satisfy homogeneity, symmetry, and curvature properties. The model is illustrated with an application to vegetable demand in Italy.

\section{Mixed Demands}

In the mixed demand setting, we consider consumers are price takers for all goods, but at the market level the prices of only a subset of goods is predetermined, whereas for the 
remaining goods it is the aggregate quantities that are predetermined. For an explicit definition of mixed demands, first introduced by Samuelson (1965) and analyzed by Chavas (1984), partition the consumption bundle into $(m+n)$ goods. Let $x \equiv\left[x_{1}, x_{2}, \ldots, x_{n}\right]$ denote the vector of commodities chosen optimally and let $z \equiv\left[z_{1}, z_{2}, \ldots, z_{m}\right]$ denote the vector of commodities in fixed quantity whose prices are optimally determined. Correspondingly, $p_{i}$ denotes the nominal price of $x_{i}$, whereas $q_{k}$ denotes the nominal price of $z_{k}$. Total consumer expenditure (income, for short) is $y$. Mixed demands are then derived from the constrained optimization problem (Samuelson 1965, p. 791):

$$
\begin{aligned}
& \max _{x, q} \quad U(x, z)-V(p, q, y) \\
& \text { s.t. } \quad p \cdot x+q \cdot z=y,
\end{aligned}
$$

where $U(\cdot)$ and $V(\cdot)$ are the direct and indirect utility functions, respectively, which are assumed quasi-concave and quasi-convex in their respective arguments, as well as satisfying standard monotonicity properties. ${ }^{2}$ The optimality conditions for an interior solution of problem (1) are:

$$
\frac{\partial U\left(x^{*}, z\right)}{\partial x_{i}}-\lambda p_{i}=0, \quad i=1, \ldots, n
$$

$$
-\frac{\partial V\left(p, q^{*}, y\right)}{\partial q_{k}}-\lambda z_{k}=0, \quad k=1, \ldots, m
$$

$$
p \cdot x^{*}+q^{*} \cdot z=y .
$$

The solutions to (2)-(4) give the Marshallian mixed demand vectors $x^{*}=x(p, z, y)$ and $q^{*}=q(p, z, y)$. Clearly, at the optimum, $U\left(x^{*}, z\right)=V\left(p, q^{*}, y\right) \equiv V^{M}(p, z, y)$, where $V^{M}(\cdot)$ is the mixed utility function.

The mixed demand functions $x(p, z, y)$ and $q(p, z, y)$ satisfy Walras's law (the adding-up condition). Moreover, the functions $x(p, z, y)$ and $q(p, z, y)$ are homogeneous of degree zero and degree one in $(p, y)$, respectively [and thus the mixed utility function is homogeneous of degree zero in $(p, y)]$. The symmetry property

\footnotetext{
${ }^{2}$ Samuelson (1965) and Chavas (1984) represent the indirect utility function in terms of normalized prices $p_{i} / y$, which helps the derivation of duality relations. As long as $y$ is given, however, the representation in (1) is admissible and simplifies the interpretation of the model.
}

applies to compensated mixed demand functions, which are the same as the compensated demands under rationing (Chavas 1984) and may be characterized in terms of the restricted cost function (Gorman 1976)

$$
C(p, z, u) \equiv \min _{x}\{p \cdot x \mid U(x, z) \geq u\} .
$$

This cost function is monotonic in its arguments, and homogeneous of degree one and concave in $p$. Also, the restricted cost function $C(p, z, u)$ is convex in $z$ if the utility function is quasiconcave (Deaton 1981). By standard duality the derivatives of this cost function yield

$$
\begin{aligned}
& \nabla_{p} C(p, z, u)=x^{h}(p, z, u) \\
& \nabla_{z} C(p, z, u)=-q^{h}(p, z, u)
\end{aligned}
$$

where $x^{h}(p, z, u)$ denote the compensated (i.e., Hicksian) mixed demands and $q^{h}(p, z, u)$ are the compensated price-dependent demand functions of $z$ (i.e., the prices that would have resulted in the quantity $z$ actually being the cost-minimizing solution). The functions $x^{h}(p, z, u)$ and $q^{h}(p, z, u)$ are homogeneous of degree zero and one in $p$, respectively, and embed standard curvature and symmetry conditions (Chavas 1984; Moschini and Vissa 1993).

\section{Coherent Specification of Mixed Demand Systems}

For the purpose of implementing the mixed demand approach, direct solution of the optimality conditions (2)-(4) of the problem in (1) is not feasible for reasonably general representations of preferences. A similar problem, of course, arises in the context of direct demand systems. The standard approach there is to specify a dual flexible representation of preferences and then apply the derivative property (i.e., the envelope theorem) to derive demand relations between observable variables that inherit, by construction, the theoretical restrictions embedded in the preference relation. For instance, upon a translog parameterization of the indirect utility function $V(p, q, y)$, direct demand equations are readily obtained via Roy's identity (Christensen, Jorgenson, and Lau 1975). A similar procedure is also available for the other polar case, when all quantities are given, whereby one can obtain an inverse demand system from a parameterization of the direct utility function via Wold's identity (Weymark 1980). 
It turns out that this procedure is of little help in the context of mixed demands. The value function for the problem in (1) is $M(p, z, y) \equiv U\left(x^{*}, z\right)-V\left(p, q^{*}, y\right)=0$, and by the envelope theorem we find (see also Samuelson 1965)

$$
\begin{gathered}
-\frac{\partial V\left(p, q^{*}, y\right)}{\partial p_{i}}-\lambda x_{i}^{*}=0, \quad i=1, \ldots, n \\
\frac{\partial U\left(x^{*}, z\right)}{\partial z_{k}}-\lambda q_{k}^{*}=0, \quad k=1, \ldots, m \\
-\frac{\partial V\left(p, q^{*}, y\right)}{\partial y}+\lambda=0 .
\end{gathered}
$$

It is apparent that the envelope theorem does not yield an explicit solution for $x^{*}$ and $q^{*}$; one still needs to solve a system of (nonlinear) equations to find the mixed demands of interest. Two related problems are apparent. First, commonly used flexible functional forms for the indirect utility function $V(p, q, y)$ do not have a closed-form solution for the direct utility function $U(x, z)$ (e.g., the translog). Second, even for more restrictive representation of preferences for which a closed-form parameterization for both $U(x, z)$ and $V(p, q, y)$ is available-as with the constant elasticitiy of substitution (CES) utility function, for example-the solution to the dual relations in (8)-(10) is not any easier than the direct solution of the optimality conditions (2)-(4) of the problem in (1) (and a solution in closed form may not be possible in either case, which is indeed what happens with the CES utility function).

Given the foregoing, and consistent with Gorman's (1976) observation that “... duality is about the choice of independent variables in terms of which to define a theory" (a point discussed further in Browning [1999]), in this article we suggest a new strategy for deriving a coherent system of mixed demands that relies on the restricted cost function $C(p, z, u)$. Our starting point is to note that Marshallian mixed demands must satisfy the identities

$$
\begin{aligned}
& x(p, z, y) \equiv x^{h}\left(p, z, V^{M}(p, z, y)\right) \\
& q(p, z, y) \equiv q^{h}\left(p, z, V^{M}(p, z, y)\right)
\end{aligned}
$$

Functions $x^{h}(p, z, u)$ and $q^{h}(p, z, u)$ can be derived for a parametric specification of $C(p, z, u)$ that satisfies the theoretical properties outlined earlier, as in equations (6)-(7). Thus, for the strategy of equations (11) and
(12) to be viable it is necessary to be able to retrieve the mixed utility function $V^{M}(p, z, y)$ from the postulated restricted cost function $C(p, z, u)$.

As noted earlier, the distinguishing feature of mixed demands (as compared with rationed demands) is that no disequilibrium occurs (goods in fixed supply are priced at their shadow value). It follows that the total cost of achieving utility level $u$, given $(p, z)$, is

$$
\begin{gathered}
C^{M}(p, z, u) \equiv C(p, z, u) \\
-\nabla_{z} C(p, z, u) \cdot z .
\end{gathered}
$$

Equation (13) defines the mixed cost function $C^{M}(p, z, u)$, so that the mixed utility function needed to implement equations (11) and (12) satisfies the identity

$$
C^{M}\left(p, z, V^{M}(p, z, y)\right) \equiv y .
$$

Then, in principle, the mixed utility $V^{M}(p, z, y)$ may be obtained by inverting (14), and that, together with (11) and (12), yields a coherent system of mixed demand equations.

The remaining stumbling block is that it is not possible to obtain a closed-form solution for $V^{M}(p, z, y)$ for an arbitrary specification of $C(p, z, u)$. For this reason, for example, the PIGLOG cost function used by Deaton (1981) to model rationed demand is not particularly appealing in the present context. The final step in our modeling strategy, therefore, is to identify a class of cost functions that allows explicit solution of the mixed utility function from the restricted cost function. Specifically, we focus on restricted cost functions that are affine in $u$, written as

$$
C(p, z, u)=F(p, z)+G(p, z) u .
$$

This form is reminiscent of the unrestricted expenditure function that Blackorby, Primont, and Russell (1978) called the Gorman Polar Form, which corresponds to quasi-homothetic preferences and has important applications for the problem of aggregating individual demand functions into market demands. Here, however, because the linear-in-utility property pertains to the restricted expenditure functions (and not to the unrestricted one), the structure in (15) does not correspond to quasihomothetic preferences. But, because this restricted cost function is linear in $u$, the shadow prices $q^{h}(p, z, u)$ will also be linear in $u$, so that by equation (13) the mixed cost function is linear in $u$ as well, implying that the identity (14) can 
in fact be solved for the mixed utility function of interest. Specifically,

$$
\begin{aligned}
& V^{M}(p, z, y) \\
& \quad=\frac{y-F(p, z)+\nabla_{z} F(p, z) \cdot z}{G(p, z)-\nabla_{z} G(p, z) \cdot z} .
\end{aligned}
$$

Before proceeding, we note that the linear homogeneity property of $C(p, z, u)$ implies that $F(p, z)$ and $G(p, z)$ are both homogeneous of degree plus one in $p$. Furthermore, assuming (without loss of generality) that $u \geq 0$, then the concavity of $C(p, z, u)$ in $p$ is ensured if both $F(p, z)$ and $G(p, z)$ are concave in $p$. Likewise, the convexity of $C(p, z, u)$ in $z$ is ensured if both $F(p, z)$ and $G(p, z)$ are convex in $z$.

In conclusion, given a restricted cost function with the structure of equation (15), equations (11) and (12) yield the mixed demand system

$$
\begin{gathered}
x(p, z, y)=\nabla_{p} F(p, z)+\nabla_{p} G(p, z) \\
\times\left[\frac{y-F(p, z)+\nabla_{z} F(p, z) \cdot z}{G(p, z)-\nabla_{z} G(p, z) \cdot z}\right] \\
-q(p, z, y)=\nabla_{z} F(p, z)+\nabla_{z} G(p, z) \\
\times\left[\frac{y-F(p, z)+\nabla_{z} F(p, z) \cdot z}{G(p, z)-\nabla_{z} G(p, z) \cdot z}\right] .
\end{gathered}
$$

Hence, for any given parameterization for the functions $F(p, z)$ and $G(p, z)$, equations (17) and (18) allow the derivation of integrable Marshallian mixed demand functions. The remaining task is that of choosing a parameterization for $F(p, z)$ and $G(p, z)$ that is sufficiently general.

\section{The NQ Parameterization}

To make the foregoing model operational, we need to parameterize the functions $F(p, z)$ and $G(p, z)$. To do so in a way that does not unduly influence estimation results, it is standard practice to ensure that the chosen parameterization satisfies the requirements of a "flexible functional form" (Diewert 1974). Several possibilities are open, but here we follow Diewert and Wales (1988) and write a NQ specification for the restricted cost function as follows:

$$
F(p, z)=\delta^{T} p+\left(a^{T} p\right)\left(\mu^{T} z\right)
$$

$$
\begin{aligned}
& G(p, z)=\beta^{T} p+\left(a^{T} p\right)\left(\gamma^{T} z\right) \\
& \quad+\frac{1}{2}\left(\frac{p^{T} B p}{a^{T} p}\right)+\frac{1}{2}\left(a^{T} p\right)\left(z^{T} \Gamma z\right) \\
& \quad+p^{T} L z,
\end{aligned}
$$

where the superscripted $T$ denotes vector transposition, $\beta$ and $\delta$ are $n \times 1$ vectors of parameters, $\gamma$ and $\mu$ are $m \times 1$ vectors of parameters, $B=\left[\beta_{i j}\right]$ is an $n \times n$ matrix of parameters, $\Gamma=\left[\gamma_{k s}\right]$ is an $m \times m$ matrix of parameters, and $L=\left[\lambda_{i k}\right]$ is an $n \times m$ matrix of parameters. All these parameters are to be estimated. On the other hand, the vector $a=\left[a_{1}, a_{2}, \ldots, a_{n}\right]^{T}$ contains arbitrarily chosen coefficients whose role is to ensure that the homogeneity property holds (recall that $F(p, z)$ and $G(p, z)$ are homogeneous of degree one in $p)$.

It can be shown that this model provides a local approximation at a point $(\bar{p}, \bar{z}, \bar{u})$ to an arbitrary cost function $C(p, z, u)$, subject to the one restriction that $\partial^{2} C / \partial u^{2}=0$ (what Diewert and Wales [1988] call the moneymetric scaling). ${ }^{3}$ Hence, the NQ form that we are proposing does satisfies the requirements of a standard flexible functional form. If (without further loss of generality) we set $\bar{p}_{i}=1$ and $\bar{z}_{k}=1$, the parameters of the model are subject to the following restrictions (Moschini and Rizzi 2007):

$$
\sum_{i=1}^{n} a_{i}=1
$$

$$
\begin{aligned}
& \beta_{i j}=\beta_{j i}, \quad \forall i, j=1, \ldots, n \\
& \gamma_{k s}=\gamma_{s k}, \quad \forall k, s=1, \ldots, m
\end{aligned}
$$

$$
\sum_{i=1}^{n} \delta_{i}=0
$$

$$
\sum_{i=1}^{n} \beta_{i}=1
$$

$$
\sum_{j=1}^{n} \beta_{i j}=0, \quad i=1, \ldots, n
$$

$$
\sum_{j=1}^{n} \lambda_{j k}=0, \quad k=1, \ldots, m .
$$

\footnotetext{
${ }^{3}$ Explicit derivation of this property is provided in Moschini and Rizzi (2007)
} 
From the NQ specification of equations (19) and (20), the explicit form of the mixed demands in equations (17) and (18) can be written as

$$
\begin{aligned}
x_{i}^{*}= & \delta_{i}+\left(\mu^{T} z\right) a_{i} \\
& +\left\{\beta_{i}+\sum_{j=1}^{n} \frac{\beta_{i j} p_{j}}{\left(a^{T} p\right)}+\sum_{k=1}^{m} \lambda_{i k} z_{k}+a_{i}\right. \\
& \left.\times\left[\gamma^{T} z-\frac{1}{2} \frac{p^{T} B p}{\left(a^{T} p\right)^{2}}+\frac{1}{2} z^{T} \Gamma z\right]\right\} \\
& \times V^{M}, \quad i=1, \ldots, n
\end{aligned}
$$

(29)

$$
\begin{aligned}
-q_{k}^{*}= & \left(a^{T} p\right) \mu_{k} \\
& +\left[\left(a^{T} p\right) \gamma_{k}+\left(a^{T} p\right) \sum_{s=1}^{m} \gamma_{k s} z_{s}\right. \\
& \left.+\sum_{j=1}^{n} \lambda_{j k} p_{j}\right] \cdot V^{M}, \quad k=1, \ldots, m,
\end{aligned}
$$

where $V^{M}$ is the mixed utility function in (16) for the particular NQ parameterization of $C(p, z, u)$ chosen, that is,

$$
V^{M}=\frac{y-\delta^{T} p}{\beta^{T} p+\frac{1}{2} \frac{p^{T} B p}{\left(a^{T} p\right)}-\frac{1}{2}\left(a^{T} p\right) z^{T} \Gamma z}
$$

For the purpose of estimation, it is desirable to express the mixed demand system in terms of expenditure shares. To this end, define

$$
\begin{aligned}
& w_{i}^{*} \equiv \frac{p_{i} x_{i}^{*}}{y}, \quad i=1, \ldots, n \\
& w_{k}^{*} \equiv \frac{q_{k}^{*} z_{k}}{y}, \quad k=1, \ldots, m
\end{aligned}
$$

so that $\sum_{i=1}^{n} w_{i}^{*}+\sum_{k=1}^{m} w_{k}^{*}=1$. Equations (31) and (32), with $x_{i}^{*}$ and $q_{k}^{*}$ as given in (28) and (29), with $V^{M}$ given by (30), and subject to the parametric restrictions (21)-(27), represent the structural estimating equations of the NQ mixed demand system.

\section{An Illustration}

The NQ mixed demand system derived here is illustrated with an application to the demand for vegetables using Italian monthly data obtained courtesy of the Italian statistical institute ISMEA (Istituto di Servizi per il Mercato Agricolo Alimentare). As part of its monitoring efforts on food consumption patterns, ISMEA maintains an extensive household data collection system (the "Panel famiglie") in partnership with ACNielsen. This effort is based on records of purchases made by a sample of 6,000 Italian households. The sample was meant to be a representative sample, stratified according to sociodemographic and location variables. Data of interest are recorded through the "home scanning" technology: every household in the sample is provided with a computer equipped with an optical scanner, which is used to record consumption information as soon as the purchased product enters the home. Data recorded in this fashion are supplemented by additional information concerning the purchase through a computerguided questionnaire, and a procedure exists to record comparable information for items without a bar code. Such elementary purchase data are electronically retrieved from each household on a weekly basis and are then aggregated for four-week intervals.

The data used here, extracted from that databank, concern consumption of vegetables aggregated at the Italian national level for the period January 1997 to April 2004. For each individual vegetable product, we observe the total expenditure (measured in $€ 1,000$ ), the quantity (measured in tons), and the price (i.e., the unit cost, measured in euro/kg). The original 95 four-week observations were reduced to eighty-eight monthly observations by averaging observations provided in the same calendar month. The long list of individual items was aggregated into the following nine products: (1) tomatoes, (2) eggplants, (3) zucchini, (4) bell peppers, (5) lettuce and chicory (including radicchio), (6) other vegetables (including fennel, carrots, asparagus, broccoli, artichokes, cauliflowers, cucumbers, onions, spinach, cabbage), (7) legumes (beans, green beans, and peas), (8) frozen vegetables, and (9) canned vegetables.

Table 1 reports some descriptive statistics for these data (mean prices are given for two distinct subperiods, for later use). It is apparent that prices, and more so the quantities of fresh vegetables, are affected by strong fluctuations due to seasonal variations in both demand and supply. Italian consumers value the quality of fresh vegetables, and thus favor products that are "in season," which are predominantly 
Table 1. Descriptive Statistics of Data (Monthly Observations, January 1997 to April 2004)

\begin{tabular}{|c|c|c|c|c|c|c|c|}
\hline \multirow[b]{2}{*}{ Product } & \multicolumn{4}{|c|}{ Prices (euro/kg) } & \multicolumn{3}{|c|}{ Total Consumption (Tons) } \\
\hline & $\begin{array}{l}\text { Mean } \\
97-01\end{array}$ & $\begin{array}{l}\text { Mean } \\
02-04\end{array}$ & Min. & Max. & Mean & Min. & Max. \\
\hline 1. Tomatoes & 1.45 & 1.81 & 0.80 & 2.37 & 23,792 & 8,821 & 49,716 \\
\hline 2. Eggplants & 1.25 & 1.48 & 0.72 & 2.69 & 7,162 & 1,984 & 14,587 \\
\hline 3. Zucchini & 1.49 & 1.84 & 0.96 & 3.44 & 9,609 & 3,378 & 17,117 \\
\hline 4. Bell peppers & 1.66 & 1.91 & 1.08 & 2.56 & 7,385 & 3,369 & 15,559 \\
\hline 5. Lettuce and chicory & 1.53 & 1.83 & 1.25 & 2.73 & 19,798 & 10,739 & 26,202 \\
\hline 6. Other vegetables & 1.23 & 1.46 & 1.10 & 1.79 & 65,679 & 19,704 & 99,933 \\
\hline 7. Legumes & 2.02 & 2.31 & 1.40 & 3.06 & 5,583 & 1,016 & 14,706 \\
\hline 8. Frozen vegetables & 3.13 & 3.35 & 2.84 & 3.67 & 13,336 & 5,940 & 22,144 \\
\hline 9. Canned vegetables & 0.85 & 1.02 & 0.71 & 1.30 & 67,742 & 35,790 & 85,589 \\
\hline Vegetables total expenditure $(€ 1,000)$ & & & & & 298,697 & 190,248 & 359,791 \\
\hline
\end{tabular}

locally produced. Our presumption, therefore, is that the supplies of fresh vegetables (goods 1 to 7 as per the above aggregation) are predetermined and perishable, so that prices adjust to clear the market for these goods. On the other hand, the last two goods (frozen vegetables and canned vegetables) are easily stored, and we treat their prices as given in the consumer problem. Thus, as in Barten (1992), this data set appears to fulfill the basic assumptions underlying the applicability of a mixed demand system. Finally, to apply our mixed demand system to this subset of goods at the aggregate level, we also postulate that the representative consumer's preferences are weakly separable in the appropriate partition (Blackorby, Primont, and Russell 1978).

\section{Results}

The conditional mixed demand system to be estimated is composed of nine equations, seven of which have the form of equation (29) and two of which have the form of equation (28), where $y$ is the total expenditure on the nine goods. Prior to estimation, quantities $z_{k}$ and prices $p_{i}$ were normalized so as to have unit mean. Furthermore, the aggregator $\left(a^{T} p_{t}\right)=$ $\sum_{i=1}^{2} a_{i} p_{i t}$ was constructed as a Laspeyres index (i.e., with the weights $a_{i}$ set to equal average shares of the two goods involved). As discussed earlier, the NQ system is estimated in its share representation, as in equations (31) and (32), with $x_{i}^{*}$ and $q_{k}^{*}$ as in equations (28) and (29), with $V^{M}$ given by (30), and subject to the parametric restrictions (21)-(27), where $n=2$ and $m=7$. Given that $\sum_{i=1}^{n} w_{i}^{*}+$ $\sum_{k=1}^{m} w_{k}^{*}=1$, this constitutes a singular system, and therefore one equation is dropped prior to estimation.
The stochastic form of the estimating system can be represented as

$$
w_{t}=f\left(X_{t}, \theta\right)+e_{t}, \quad t=1, \ldots, 88,
$$

where $w_{t}=\left(w_{1 t}, \ldots, w_{8 t}\right)^{T}$ is the vector of $(m+n-1)$ budget shares for observation $t$; $f(\cdot)$ is the vector-value function as per the NQ structure derived earlier, with $X_{t}$ representing the vector of all explanatory variables for observation $t$ and $\theta$ denoting the vector of all parameters to be estimated; and $e_{t}=\left(e_{1 t}, \ldots, e_{8 t}\right)^{T}$ is the error vector. Assuming that this error vector is multinormally distributed with zero mean and a constant contemporaneous covariance matrix allows maximum likelihood (ML) estimation (e.g., Davidson and MacKinnon 1993, Chapter 9), which is invariant with respect to which equation is omitted.

Preliminary analysis, however, suggested the need to consider two additional issues: seasonality, and serial correlation in the estimated residuals. To handle seasonality-a standard feature of empirical studies that (like ours) rely on monthly data-we seasonally adjusted the price and quantity data prior to estimating the demand system by using monthly dummy variables, following the procedure discussed in Davidson and MacKinnon (1993, Section 19.6). To account for serial correlation, we postulated a first-order autocorrelation process and therefore estimate the following model:

$$
\begin{aligned}
w_{t}= & f\left(X_{t}, \theta\right)+R \cdot\left[w_{t-1}-f\left(X_{t-1}, \theta\right)\right] \\
& +\varepsilon_{t}, \quad t=2, \ldots, 88
\end{aligned}
$$

where $R=\rho I$, and where all variables are now seasonally adjusted. Note that the autocorrelation coefficient $\rho$ is constrained to be the 
Table 2. Summary Results for Estimated NQ Mixed Demand System

\begin{tabular}{lccccc}
\hline & \multicolumn{2}{c}{ Share Equations } & & \multicolumn{2}{c}{$\begin{array}{c}\text { Price/Quantity } \\
\text { Equations }\end{array}$} \\
\cline { 2 - 3 } \cline { 5 - 6 } Equations of & $\bar{R}^{2}$ & $D W$ & & $\bar{R}^{2}$ & $D W$ \\
\hline 1. Tomatoes & 0.42 & 1.80 & & 0.81 & 1.77 \\
2. Eggplants & 0.35 & 2.48 & & 0.65 & 2.50 \\
3. Zucchini & 0.27 & 1.71 & & 0.82 & 1.68 \\
4. Bell peppers & 0.58 & 1.87 & & 0.75 & 1.88 \\
5. Lettuce and chicory & 0.70 & 1.58 & & 0.87 & 1.61 \\
6. Other vegetables & 0.80 & 1.64 & & 0.94 & 1.62 \\
7. Legumes & 0.88 & 2.15 & & 0.73 & 2.06 \\
8. Frozen vegetables & 0.58 & 2.03 & & 0.65 & 2.07 \\
9. Canned vegetables & 0.48 & 1.75 & & 0.66 & 1.80 \\
Log likelihood & 2,916 & & & & \\
Number of parameters & 53 & & & & \\
Observations per equation & 87 & & & & \\
Autocorrelation coefficient & $\hat{\rho}=0.5058( \pm 0.0329)$ & & \\
\hline
\end{tabular}

same for all equations, which provides the simplest structure guaranteeing that the resulting stochastic system satisfies adding-up (Berndt and Savin 1975). The standard assumptions leading to ML estimation, discussed earlier, now apply to the vector $\varepsilon_{t}$.

ML estimation of this system was carried out by using the software package TSP (version 4.5). Some estimation results are reported in table 2. We find that the proposed model yields a satisfactory fit, as measured by the $R^{2}$ of the individual equations. A reviewer expressed concern about the relatively low fit of some share equations. In table 2 we report the $R^{2}$ of the implied estimate of the price and quantity equations underlying the share equations to illustrate that the possible low fit is, to a certain extent, a reflection of measuring it from the share equation perspective. The estimated autocorrelation coefficient $(\hat{\rho}=0.51)$ is significantly different from zero. The DurbinWatson statistics for the individual equations of table 2 also suggest that serial correlation is satisfactorily handled by the simple first-order structure implemented in (34).

Detailed estimation results for the individual parameters are omitted here (they are available upon request). Instead, below we report the elasticity values implied by the estimated parameters, which are more directly informative of the underlying demand structure. But we note that the underlying restricted cost function, once evaluated at the estimated parameter values, satisfies the curvature properties implied by theory. Specifically, the estimated restricted cost function turns out to be convex in $z$ (globally so, because the Hessian in question entails only constant parameters) and concave in $p$ (at the point $p_{i}=z_{k}=y=1$ ).

\section{Elasticities}

As with standard demand analysis, the sensitivity of the endogenous variables to changes in predetermined variables is best illustrated by elasticities. In our setting, given the presumption that the monthly quantities of fresh vegetables are best viewed as predetermined at the aggregate level, interest centers on the Marshallian mixed elasticities that are directly derived from our estimated equations. Table 3 reports the estimated own-quantity and ownprice Marshallian mixed elasticities, and the expenditure elasticities, evaluated at the sample mean of the predetermined variables $\left(p_{i}=\right.$ $\left.z_{k}=y=1\right)$. For these Marshallian elastities we also report standard errors (in parentheses, under the corresponding elasticity) computed using the standard delta method. ${ }^{4}$ As expected, given that the restricted cost function satisfies the curvature conditions, the coefficients of own-quantity and own-price effects are negative. The fact that the own-quantity mixed elasticities of the price equations are negative and less than one in absolute value suggests that the ordinary demand for vegetables is elastic

\footnotetext{
${ }^{4}$ The elasticities in Table 3 were computed by taking analytic derivates of the mixed demand equations and, as per the appropriate elasticity definition, by using the TSP command DIFFER and then evaluated at the estimated parameter values and at the chosen point of the explanatory variables. In addition, the TSP command ANALYZ allowed calculation of the asymptotic standard errors of the estimated elasticities (a nonlinear combination of random variables) through the implementation of the so-called delta method (Hall and Cummins 1999).
} 
Table 3. Marshallian Mixed Demand Elasticities (Evaluated at the Sample Mean)

\begin{tabular}{lll}
\hline & Own Quantity or & \\
\hline$q_{1}$ & $-0.54(0.1071)$ & Expenditure \\
$q_{2}$ & $-0.59(0.1032)$ & $1.14(0.1246)$ \\
$q_{3}$ & $-0.87(0.1236)$ & $1.28(0.1581)$ \\
$q_{4}$ & $-0.48(0.0663)$ & $1.14(0.1730)$ \\
$q_{5}$ & $-0.39(0.0836)$ & $1.30(0.1009)$ \\
$q_{6}$ & $-0.34(0.0351)$ & $0.86(0.0556)$ \\
$q_{7}$ & $-0.27(0.0364)$ & $1.15(0.1208)$ \\
$x_{1}$ & $-0.65(0.0876)$ & $0.83(0.1041)$ \\
$x_{2}$ & $-0.74(0.0914)$ & $0.93(0.1100)$ \\
\hline
\end{tabular}

Note: See table 4 for the indices of variables $q_{i}$ and $x_{k}$.

with respect to own price. On the other hand, the estimated values for the own-price elasticities of the quantity equations, also less than one in absolute value, indicate a relatively inelastic demand for both frozen vegetables and canned vegetables. ${ }^{5}$

The question of substitution and/or complementarity relations between the goods is here best posed in terms of the mixed Hicksian (compensated) elasticities, defined as follows:

$$
\begin{aligned}
\theta_{k s}^{h} & =\left(\partial q_{k}^{h}(p, z, u) / \partial z_{s}\right)\left(z_{s} / q_{k}\right), \\
k, s & =1, \ldots, 7 \\
\rho_{k i}^{h} & =\left(\partial q_{k}^{h}(z, p, u) / \partial p_{i}\right)\left(p_{i} / q_{k}\right), \\
k & =1, \ldots, 7 ; \quad i=1,2 \\
\psi_{i k}^{h} & =\left(\partial x_{i}^{h}(z, p, u) / \partial z_{k}\right)\left(z_{k} / x_{i}\right), \\
i & =1,2 ; \quad k=1, \ldots, 7 \\
\eta_{i j}^{h} & =\left(\partial x_{i}^{h}(z, p, u) / \partial p_{j}\right)\left(p_{j} / x_{i}\right), \\
i, j & =1,2 .
\end{aligned}
$$

The curvature properties of the restricted cost function (concavity in $p$ and convexity in $z$ ) would imply that own-price and own-quantity elasticities, $\eta_{i i}^{h}$ and $\theta_{k k}^{h}$, are nonpositive. Furthermore, the compensated cross elasticities can characterize the substitutability pattern among goods. Specifically, the so-called $R$ classification ( $R$ for "rationed") proposed by Madden (1991) is of interest here. According to this classification, quantity constrained goods

\footnotetext{
${ }^{5}$ These observations, of course, must be interpreted in the mixes demand framework. If interest were on the usual Marshallian elasticities of direct demand systems, then such elasticities could be computed as outlined in Moschini and Vissa (1993).
}

$z_{k}$ and $z_{s}$ are substitutes if $\theta_{k s}^{h}<0$ and complements if $\theta_{k s}^{h}>0$; unconstrained goods $x_{i}$ and $x_{j}$ are substitutes if $\eta_{i j}^{h}>0$ and complements if $\eta_{i j}^{h}<0$; unconstrained goods $x_{i}$ and constrained goods $z_{k}$ are substitutes if $\rho_{k i}^{h}>0$ and $\psi_{i k}^{h}<0$, whereas they are complements when $\rho_{k i}^{h}<0$ and $\psi_{i k}^{h}>0$.

The complete matrix of compensated mixed elasticities, evaluated at the mean point of the data, is reported in table 4. As for the Marshallian elasticities of table 3 , we computed the standard errors by the delta method, but for clarity they are omitted from table 4 . We note, however, that most of these elasticities ( 75 out of 81 estimates, including all of the diagonal elements) are significant at the 5\% level. The own-price and own-quantity effects are negative, as implied by the curvature property of the restricted cost function (which was not explicitly maintained but turned out to be satisfied by the estimated parameters). The most inelastic Hicksian demand is that for canned vegetables (own-price elasticity of -0.21) and the most elastic Hicksian demand is that for zucchini (own-quantity elasticity of -1.10). Furthermore, it is apparent that the goods we are modeling are all $R$-substitutes, as per Madden's (1991) definition discussed in the preceding paragraph. Specifically, within the fresh vegetables group we have $\theta_{k s}^{h}<0$; within the processed vegetables group we have $\eta_{i j}^{h}>0$; and between fresh and processed vegetables we find $\rho_{k i}^{h}>0$ and $\psi_{i k}^{h}<0$. Lettuce and radicchio, and other vegetables, appear to have the strongest substitution effect with the remaining five fresh vegetables categories. Frozen and canned vegetables also appear to be effective substitutes for fresh vegetables.

\section{The Euro Effect}

As a final application of the empirical illustration of the NQ model proposed in this article, we consider the lively debate that has recently emerged in Europe, and especially in Italy, on the inflationary effects of the introduction of the euro in January 2002. The question is whether the "changeover" was accompanied by an unexpected increase in prices, possibly imperfectly measured by official statistics, or whether consumers' perception of the price increases were, simply put, at variance with the facts (e.g., Marini, Piergallini, and Scaramozzino 2004). The sample period of our data encompasses the date of the introduction 
Table 4. Hicksian Mixed Demand Elasticities (Evaluated at the Sample Mean)

\begin{tabular}{rccccccccc}
\hline & $z_{1}$ & $z_{2}$ & $z_{3}$ & $z_{4}$ & $z_{5}$ & $z_{6}$ & $z_{7}$ & \multicolumn{1}{c}{$p_{1}$} & $p_{2}$ \\
\hline$q_{1}$ & -0.96 & -0.12 & -0.13 & -0.19 & -0.54 & -0.89 & -0.15 & 0.32 & 0.68 \\
$q_{2}$ & -0.48 & -0.70 & -0.29 & -0.09 & -0.75 & -0.87 & -0.10 & 0.53 & 0.47 \\
$q_{3}$ & -0.32 & -0.18 & -1.10 & -0.02 & -0.63 & -1.09 & -0.20 & 0.48 & 0.52 \\
$q_{4}$ & -0.57 & -0.06 & -0.02 & -0.62 & -0.53 & -0.90 & -0.17 & 0.34 & 0.66 \\
$q_{5}$ & -0.64 & -0.23 & -0.30 & -0.22 & -0.89 & -1.20 & -0.22 & 0.51 & 0.49 \\
$q_{6}$ & -0.40 & -0.10 & -0.20 & -0.14 & -0.45 & -0.88 & -0.13 & 0.43 & 0.57 \\
$q_{7}$ & -0.47 & -0.08 & -0.25 & -0.18 & -0.60 & -0.88 & -0.40 & 0.58 & 0.42 \\
$x_{1}$ & -0.29 & -0.12 & -0.18 & -0.10 & -0.39 & -0.88 & -0.16 & -0.29 & 0.29 \\
$x_{2}$ & -0.43 & -0.08 & -0.13 & -0.15 & -0.27 & -0.81 & -0.09 & 0.21 & -0.21 \\
\hline
\end{tabular}

Note: Each entry is the elasticity of the row variable with respect to the column variable. Indices for $(q, z)$ variables: $1=$ tomatoes, $2=$ eggplants, $3=$ zucchini, $4=$ bell peppers, $5=$ lettuce and chicory, $6=$ other fresh vegetables, $7=$ legumes. Indices for $(p, x)$ variables: $1=$ frozen vegetables, $2=$ canned vegetables.

of the euro, and the price behavior of the goods in our bundles does show some upward trend following the introduction of the euro (table 1).

Because our model is largely a pricedetermination model (for seven of the nine goods the assumption is that supplies in any given month are given, and the corresponding prices adjust to clear the market), the hypothesis of an inflationary effect of the euro introduction suggests that we test whether our model supports a structural change occurring with the introduction of the euro. Specifically, we focus on a possible structural break occurring in January 2002, when the European common currency (the euro) replaced the lira. The specific test that we consider is based on the statistic

$$
\Lambda=2\left[\frac{T}{T_{1}} L_{1}^{*}+\frac{1}{2} \log \frac{T}{T_{1}}-L^{*}\right]
$$

where $T=87$ and $T_{1}=59$ are the sizes of the full sample and of the sub-sample up to the hypothesized structural break (i.e., February 1997 to December 2001), $L^{*}$ is the maximized value of the log-likelihood function over the entire sample, and $L_{1}^{*}$ is the maximized value of the log-likelihood function over the subsample of $T_{1}$. We find that the computed value of this statistic is $\Lambda=184$.1. Under the null hypothesis of parameter stability, the statistic $\Lambda$ is distributed as $\chi^{2}$ with $\left(T-T_{1}\right) N=224 \mathrm{de}$ grees of freedom, where $N=8$ is the number of estimating equations (Anderson and Blundell 1984). Thus, we find no structural change: the hypothesis of constancy of the parameters after the introduction of the euro is not rejected at the customary 5\% significance level.

\section{Conclusions}

This article has dealt with the specification of a flexible and theory-consistent system of Samuelson's (1965) mixed demand functions. This approach allows for a richer set of possibilities about what is to be assumed as exogenous so that the identifying assumptions of demand models can be tailored to the nature of the data at hand. The paucity of existing applications of mixed demand systems in empirical demand analysis is possibly due to the fact that derivation of theory-consistent mixed demand equations is somewhat more difficult than for ordinary demand equations. After discussing this problem in some detail, we suggested a new approach to specifying a coherent mixed demand system based on the restricted expenditure function typically studied in the related area of rationed demands. The approach was implemented by a proposed new NQ parameterization of this restricted cost function. We showed that this formulation is locally flexible in the sense of being able to provide a secondorder approximation to an arbitrary mixed cost function.

The new NQ mixed demand system was illustrated with an application to the Italian demand for vegetables. Specifically, we estimated a nine-good mixed demand system for the group of fresh and processed vegetables, whereby we allow for seven goods (categories of fresh vegetables) to be represented by predetermined supply, with price adjusting to clear the market, and for two goods (canned and frozen vegetables) to have the standard representation (prices are given). The system was estimated with monthly data obtained from a large representative and 
stratified sample of Italian households. The fit of the estimated equations is fairly good and the one-parameter correction for serial correlation seems to work well. The estimated elasticities appear plausible and conform to Madden's (1991) notion of $R$ substitutes, as perhaps one should have expected. This application illustrates that the new system that we have derived is readily estimable and can be quite useful for modeling demand in markets in which some prices are exogenously given, whereas other prices are endogenously determined given quantities supplied.

\section{[Received April 2006; accepted January 2007.]}

\section{References}

Amemiya, Y. 1985. "Instrumental Variable Estimator for the Nonlinear Errors-in-Variables Model." Journal of Econometrics 28:273-289.

Anderson, G., and R. Blundell. 1984. "Consumer Non-durables in the U.K.: A Dynamic Demand System." Economic Journal, Conference Papers 94:34-44.

Banks, J., R. Blundell, and A. Lewbel. 1997. "Quadratic Engel Curves and Consumer Demand." Review of Economics and Statistics 79:527-539.

Barten, A.P. 1992. "The Estimation of Mixed Demand Systems." In R. Bewley and Tran Van Hoa, eds. Contributions to Consumer Demand and Econometrics: Essays in Honour of Henri Theil. London: Macmillan, pp. 31-57.

Barten, A.P., and L.J. Bettendorf. 1989. "Price Formation of Fish: An Application of an Inverse Demand System." European Economic Review 33:1509-1525.

Berndt, E.R., and N.E. Savin. 1975. "Estimation and Hypothesis Testing in Singular Equation Systems with Autoregressive Disturbances." Econometrica 43:937-957.

Blackorby, C., D. Primont, and R.R. Russell. 1978. Duality, Separability, and Functional Structure: Theory and Economic Applications. New York: North-Holland.

Brown, M.G., and J.Y. Lee. 2006. "A Rotterdam Mixed Demand System." In M. Holt and J.P. Chavas, eds. Essays in Honor of Stanley R. Johnson. Berkeley Electronic Press, Article 14. Available at http://www.bepress.com/ sjohnson/art14/.

Browning, M. 1999. "Dual Approaches to Utility." In S. Barbera, P.J. Hammond, and C. Seidl, eds. Handbook of Utility Theory: Vol. 1: Principles. Dordrecht: Kluwer Academic, pp. 123-141.
Chavas, J-P. 1984. "The Theory of Mixed Demand Functions." European Economic Review 24:321-344.

Christensen, L.R., D.W. Jorgenson, and L.J. Lau. 1975. "Transcendental Logarithmic Utility Functions." American Economic Review 65:367-383.

Cunha-e-Sá, M.A., and M.M. Ducla-Soares. 1999. "Specification Tests for Mixed Demand Systems with an Emphasis on Combining Contingent Valuation and Revealed Data." Journal of Environmental Economics and Management 38:215-233.

Davidson, R., and J.G. MacKinnon. 1993. Estimation and Inference in Econometrics. Oxford: Oxford University Press.

Deaton, A. 1981. "Theoretical and Empirical Approaches to Consumer Demand under Rationing." In A. Deaton, ed. Essays in the Theory and Measurement of Consumer Behaviour in Honour of Sir Richard Stone. Cambridge: Cambridge University Press, pp. 55-72.

Deaton, A., and J. Muellbauer. 1980. "An Almost Ideal Demand System." American Economic Review 70:312-326.

Diewert, W.E. 1974. "Applications of Duality Theory." In M.D. Intriligator and D.A. Kendrick, eds. Frontiers of Quantitative Economics, vol. II. Amsterdam: North-Holland, pp. 106-171.

Diewert, W.E., and T.J. Wales. 1988. "Normalized Quadratic Systems of Consumer Demand Functions." Journal of Business \& Economic Statistics 6:303-312.

Eales, J.S., and L.J. Unnevehr. 1994. "The Inverse Almost Ideal Demand System." European Economic Review 38:101-115.

Geary, R.C. 1949-1950. "A Note on 'A Constant Utility Index of the Cost of Living." Review of Economic Studies 18:65-66.

Gorman, W.M. 1976. "Tricks with Utility Functions." In M. Artis and R. Nobay, eds. Essays in Economic Analysis. Cambridge: Cambridge University Press, pp. 211-243.

Hall, B.H., and C. Cummins. 1999. Time Series Processor Version 4.5-Reference Manual. Palo Alto, CA: TSP International, April.

Holt, M.T., and R.C. Bishop. 2002. "A Semiflexible Normalized Quadratic Inverse Demand System: An Application to the Price Formation of Fish." Empirical Economics 27:2347.

Lewbel, A. 1996. "Demand Estimation with expenditure Measurement Errors on the Left and Right Hand Side." Review of Economics and Statistics 78:718-725.

Madden, P. 1991. "A Generalization of Hicksian q Substitutes and Complements with 
Application to Demand Rationing." Econometrica 59:1497-1508.

Marini, G., A. Piergallini, and P. Scaramozzino. 2004. "Inflation Bias after the Euro: Evidence from the UK and Italy." Working Paper, Department of Economics, University of Rome "Tor Vergata."

Matsuda, T. 2004. "Incorporating Generalized Marginal Budget Shares in a Mixed Demand System." American Journal of Agricultural Economics 86:1117-1126.

Moschini, G. 1998. "The Semiflexible Almost Ideal Demand System." European Economic Review 42:349-364.

- 2001. "Production Risk and the Estimation of Ex-ante Cost Functions." Journal of Econometrics 100:357-380.

Moschini, G., and P.L. Rizzi. 2006. "Coherent Specification of a Mixed Demand System: The Stone-Geary Model." In M. Holt and J.-P. Chavas, eds. Essays in Honor of Stanley R. Johnson. Berkeley Electronic Press, Article 15. Available at http://www.bepress.com/ sjohnson/art15/.

.2007. "AJAE Appendix: Deriving a Flexible Mixed Demand System: The Normalized
Quadratic Model." Unpublished manuscript. Available at http://agecon.lib.umn.edu.

Moschini, G., and A. Vissa. 1992. "A Linear Inverse Demand System." Journal of Agricultural and Resource Economics 17:294-302.

Moschini, G., and A. Vissa. 1993. "Flexible Specification of Mixed Demand Systems." American Journal of Agricultural Economics 75:1-9.

Neary, J.P., and K.W.S. Roberts. 1980. "The Theory of Household Behavior under Rationing." European Economic Review 13:25-42.

Samuelson, P.A. 1965. "Using Full Duality to Show That Simultaneously Additive Direct and Indirect Utilities Implies Unitary Price Elasticity of Demand." Econometrica 33:781796.

Stone, J.R.N. 1954. "Linear Expenditure Systems and Demand Analysis: An Application to the Pattern of British Demand.” Economic Journal 54:511-527.

Theil, H. 1975 and 1976. The Theory and Measurement of Consumer Demand, vols. I and II. Amsterdam: North-Holland.

Weymark, J.A. 1980. "Duality Results in Demand Theory." European Economic Review 14:377395. 